\title{
On the Coupling Between the Incus and the Stapes in the Cat
}

\author{
W. Robert J. Funnell, ${ }^{1,2}$ T. Heng Siah, ${ }^{1}$ Marc D. McKee, ${ }^{3,4}$ Sam J. Daniel, ${ }^{2}$ \\ and Willem F. Decraemer ${ }^{5}$ \\ ${ }^{1}$ Department of BioMedical Engineering, McGill University, 3775, rue University, Montréal, QC, Canada H3A 2B4 \\ ${ }^{2}$ Department of Otolaryngology, McGill University, Montréal, QC, Canada H3A $2 B 4$ \\ ${ }^{3}$ Faculty of Dentistry, McGill University, Montréal, QC, Canada H3A $2 B 2$ \\ ${ }^{4}$ Department of Anatomy and Cell Biology, McGill University, Montréal, QC, Canada H3A 2B2 \\ ${ }^{5}$ Laboratory of BioMedical Physics, University of Antwerp, Antwerp, Belgium 2020
}

Received: 2 March 2004; Accepted: 1 September 2004; Online publication: 2 February 2005

\begin{abstract}
The connection between the long process and the lenticular process of the incus is extremely fine, so much so that some authors have treated the lenticular process as a separate bone. We review descriptions of the lenticular process that have appeared in the literature, and present some new histological observations. We discuss the dimensions and composition of the lenticular process and of the incudostapedial joint, and present estimates of the material properties for the bone, cartilage, and ligament of which they are composed. We present a preliminary finiteelement model which includes the lenticular plate, the bony pedicle connecting the lenticular plate to the long process, the head of the stapes, and the incudostapedial joint. The model has a much simplified geometry. We present simulation results for ranges of values for the material properties. We then present simulation results for this model when it is incorporated into an overall model of the middle ear of the cat. For the geometries and material properties used here, the bony pedicle is found to contribute significant flexibility to the coupling between the incus and the stapes.
\end{abstract}

Correspondence to: W. Robert J. Funnell • Department of BioMedical Engineering - McGill University $\cdot 3775$, rue University, Montréal, QC, Canada H3A 2B4. Telephone: (1) 514-3986739; fax: (1) 5143987461; email: robert.funnell@mcgill.ca
Keywords: incus, stapes, lenticular process, finiteelement model, mechanics, histology

\section{INTRODUCTION}

The connection between the long process and the lenticular process of the incus is extremely fine, so much so that some authors have treated the lenticular process as a separate bone. The region is important clinically as a frequent point of attachment for prostheses. It is also important in determining how sound is transmitted from the incus to the stapes. The goal of this paper is to begin to clarify the nature of the structure, and to explore the possible mechanical implications of its peculiar form.

We review descriptions of the lenticular process that have appeared in the literature, and present some new histological observations. We discuss the dimensions and composition of the lenticular process and of the incudostapedial joint, and present estimates of the material properties for the bone, cartilage, and ligament of which they are composed.

We then present a preliminary finite-element model of the coupling between the incus and the stapes in the cat. The model includes the lenticular plate, the bony pedicle connecting the lenticular plate to the long process, the head of the stapes, and the incudostapedial joint. The model has a much simplified geometry. We present simulation results for ranges of values for the material properties, and 
then present simulation results for this model when it is incorporated into an overall model of the cat middle ear. For the geometries and material properties used here, the bony pedicle is found to contribute more flexibility to the coupling between the incus and the stapes than does the actual incudostapedial joint itself. The nature of the flexibility of the pedicle appears to be significantly different from that of the joint, and may be important in understanding the mode of vibration of the stapes.

\section{PREVIOUS DESCRIPTIONS}

The lenticular process is very small and difficult to observe, and has often been either neglected or misinterpreted. Asherson (1978) said that the "fourth ossicle," the ossicle of Sylvius between the incus and stapes, was "described and depicted in all books on anatomy or otology" until around 1900. About 1650, however, some doubt was already being expressed by Browne, who wrote, "Heere you may also see a parcell of the smallest bones, the incus, malleus and stapes, and especially the fourth small bone at the beginning of the stapes if you admitt of it with Sylvius for a distinct bone" [emphasis added].

Shrapnell (1832) observed that the "most generally received opinion appears to be that it is a separate bone" and proceeded to illustrate convincingly that such is not the case. He clearly showed the bony pedicle joining the lenticular plate to the long process of the incus. The pedicle is shown as four or five times wider in one direction than in the other, and somewhat curved. Eysell (1870) presented a detailed drawing of a histological section, again clearly show-

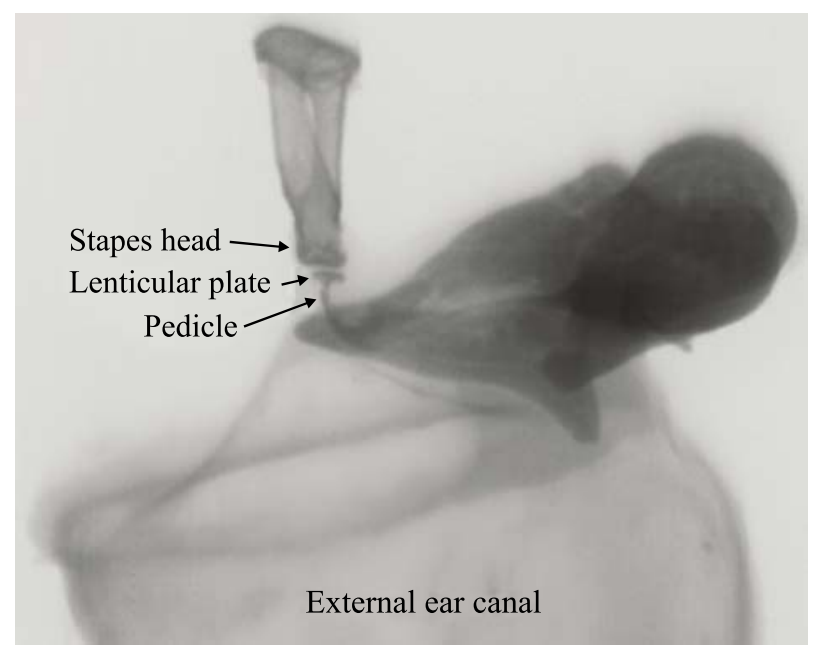

FIG. 1. High-resolution x-ray image of a total human middle-ear homograft. The image was obtained with a SkyScan 1072 microCT machine. The external ear canal is at the bottom, the heads of the malleus and incus are at the right and the stapes is at the top.

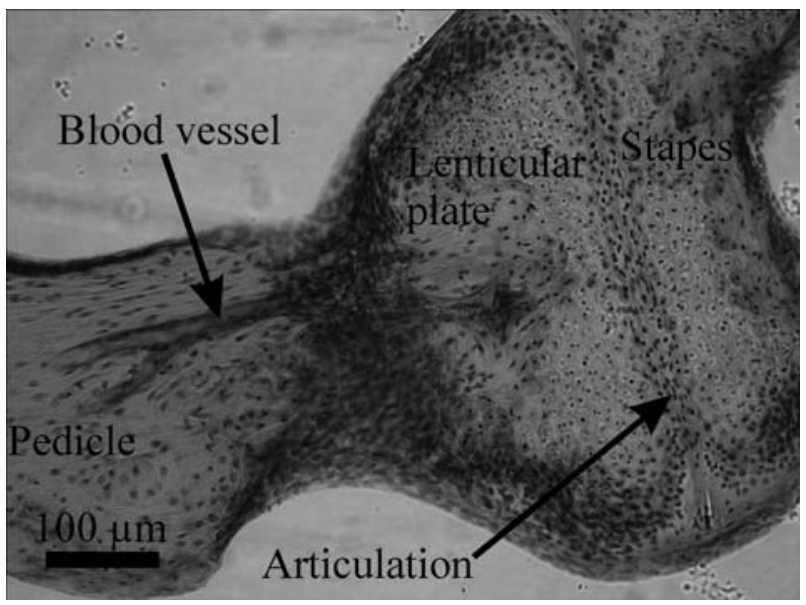

FIG. 2. A $20-\mu \mathrm{m}$ histological section from a paraffin-embedded cat ear, stained with haematoxylin and eosin, showing the pedicle and lenticular plate of the incus, the articulation between the incus and the stapes, and the head of the stapes. The plane of section is roughly parallel to the plane of the crura and perpendicular to the footplate. A blood vessel can be seen within the pedicle.

ing the continuous bony connection. Even recently, however, the lenticular process has been described as sometimes being a separate bone (e.g., Wolff et al. 1957,1971 ) or as always being so (e.g., Palchun and Magomedov 1997).

Figure 1 shows a high-resolution x-ray image (obtained with a SkyScan 1072 microCT machine) of a total human middle-ear homograft, showing the narrow dense (apparently bony) pedicle between the incus and the lenticular plate. Hüttenbrink (1997) presented a similar x-ray image but the continuity of the connection was not quite so evident.

The preceding discussion refers to the human ear. Descriptions of the lenticular process in other species have been few, and they have often been unspecific and ambiguous. Among the more detailed are those of Hyrtl (1845), Doran (1878), and Henson (1961). Hyrtl said that "the Ossiculum lenticulare Sylvii is in no animal an independent ossicle, but an apophysis of the long process of the incus." Doran referred to it as an apophysis, or perhaps epiphysis, of the incus, and cited the "beautiful description" by Eysell (1870). Henson described middle-ear structures in three insectivores and eight bats; his overall description of the lenticular process was as "a small cartilaginous disk mounted on an osseous pedicle."

\section{HISTOLOGY}

In this section, we present some histological observations of the incus and stapes in the cat. These observations form the basis of the finite-element model presented later. 


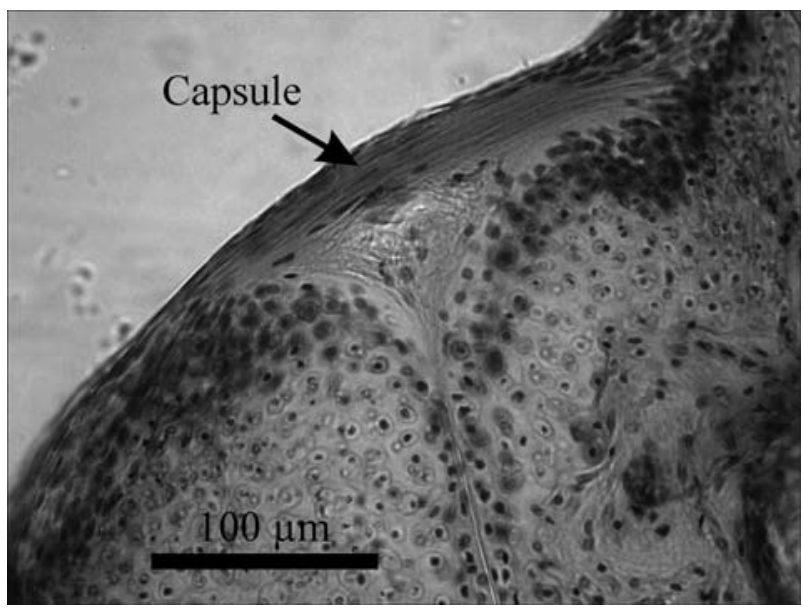

FIG. 3. Higher-magnification view of the same histological section as in Figure 2, showing the joint capsule.

Figures 2 and 3 show a $20-\mu \mathrm{m}$ histological section from a paraffin-embedded cat ear, stained with haematoxylin and eosin. Every second section was stained and mounted. Figure 2 shows the pedicle and lenticular plate of the long process of the incus, and the articulation between the incus and the stapes. This slide came the closest to showing the center of the connection between the pedicle and the lenticular plate. Because the section is so thick, and because the neighboring sections are not available, the nature of the connection between the long process and the lenticular plate cannot be clearly determined.

Figure 3, a higher-magnification view from the same section as Figure 2, shows the ligament of the

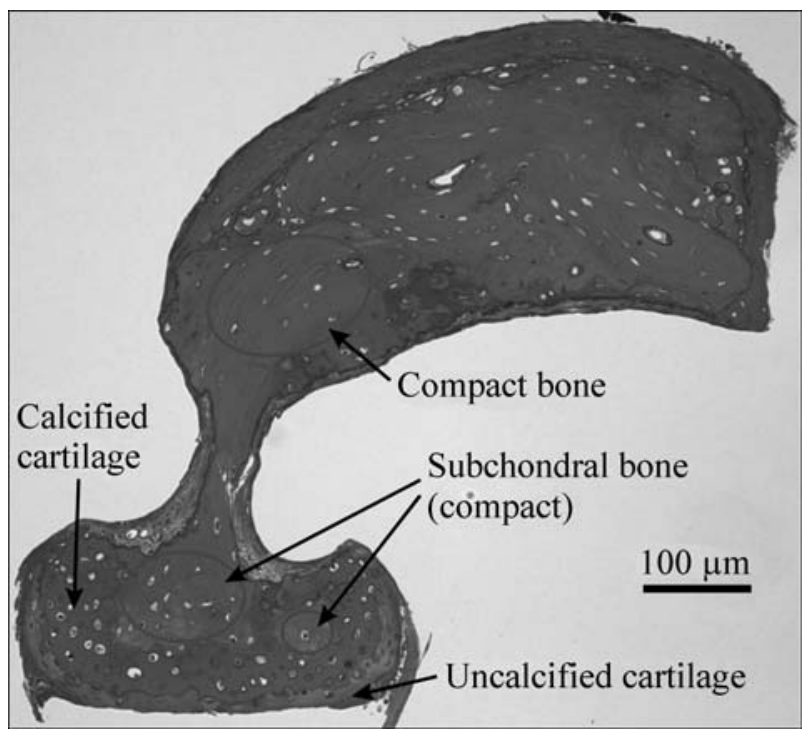

FIG. 4. A 1- $\mu \mathrm{m}$ histological section from a plastic-embedded cat incus, stained with toluidene blue, showing the tip of the long process (top right) and the lenticular plate (bottom left). The plane of section is roughly perpendicular to the articular face of the lenticular plate.

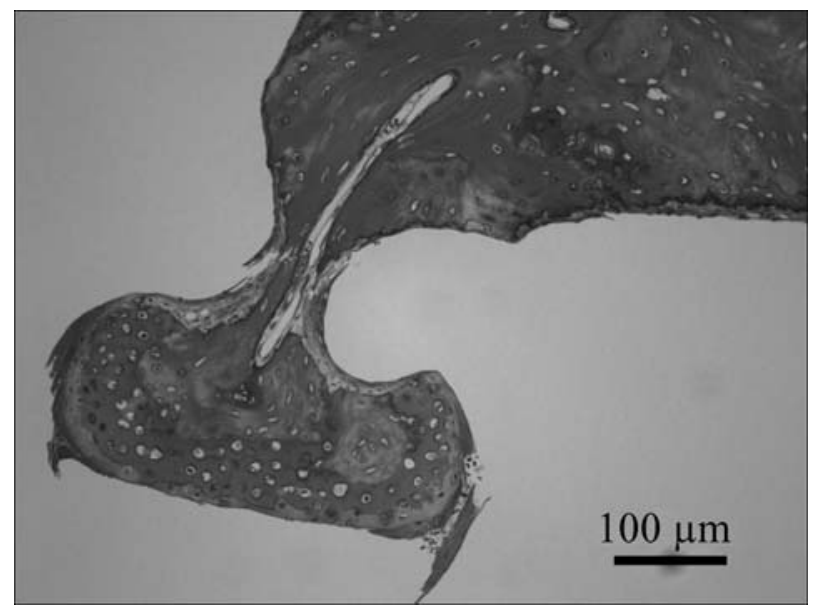

FIG. 5. A different 1- $\mu \mathrm{m}$ section from the same incus as shown in Figure 4; the two sections are $23 \mu \mathrm{m}$ apart. Two blood vessels can be seen running alongside each other between the long process and the lenticular plate.

incudostapedial joint capsule. The fibrous nature of the capsule can be clearly seen. In this figure, one can also see the extremely fine synovial space of the incudostapedial joint; the cartilaginous layers on either side of the joint are almost, if not quite, in contact.

To visualize the pedicle more clearly, we produced a new set of serial sections from a plastic-embedded incus of an adult cat. The sections were cut at a thickness of $1 \mu \mathrm{m}$, and every section was stained with toluidene blue and mounted. Figure 4 very clearly shows the pedicle as a bony connection from the long process to the lenticular plate. (The pedicle is cracked; it was presumably broken during dissection.) The lenticular plate is composed largely of calcified cartilage, with islands of subchondral ('under cartilage') compact bone. Calcified cartilage is a mineralized cartilage layer directly apposed to bone and is about 10 times stiffer than uncalcified cartilage. The articulating surface of the lenticular plate is a thin layer of uncalcified cartilage, which stains less darkly with toluidene blue. The long process of the incus consists mainly of compact bone.

Figure 5 shows a pair of blood vessels running within the pedicle, but not entirely encased in bone. A blood vessel can also be seen in the pedicle in Figure 2, although in this plane of section it appears to be entirely within the bone.

\section{FINITE-ELEMENT MODEL}

\section{Geometry}

For ease of mesh generation, our current model consists of simple blocks. The 3-D configuration of the model is shown in Figure 6. On the left the 


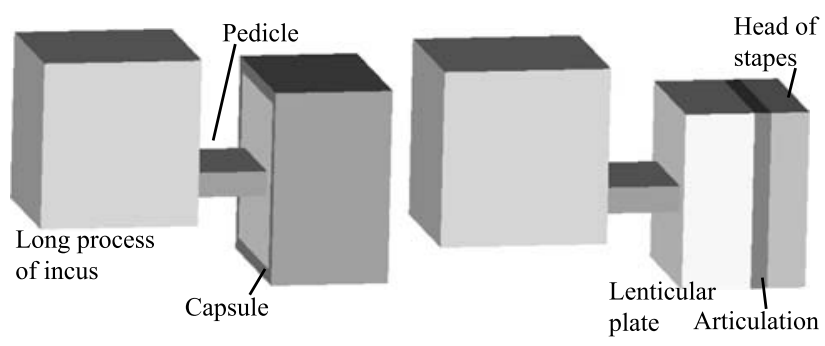

FIG. 6. 3-D block configuration of the pedicle-and-joint model. On the left the complete model is shown; on the right the joint capsule has been removed to show the underlying blocks corresponding to the lenticular plate, the articulation and the head of the stapes.

complete model is shown; on the right the joint capsule has been removed to show the underlying blocks corresponding to the lenticular plate, the articulation and the head of the stapes. The detailed dimensions are shown in Figure 7, with a side view on the left and a top view on the right. The dimensions were estimated from our cat serial histological sections, including the ones presented in Figures $2-5$. The plane of the side view in Figure 7 is approximately parallel to the plane of section in Figures 4 and 5 .

The long process of the incus is represented as a block $325 \times 350 \times 370 \mu \mathrm{m}$, corresponding to just the tip of the process. The rest of the process is assumed to be so thick that it is rigid and need not be modeled. The pedicle block is $240 \mu \mathrm{m}$ wide and 160 $\mu \mathrm{m}$ long, and only $55 \mu \mathrm{m}$ thick.

The lenticular plate and the head of the stapes are both $325 \mu \mathrm{m}$ by $325 \mu \mathrm{m}$. They are separated by a single layer, $35 \mu \mathrm{m}$ thick, which represents the joint itself, as discussed below. The joint capsule completely surrounds these three structures. As shown in Figure 8, the capsule is connected to the lenticular plate and the head of the stapes only at its ends, representing the configuration seen in Figure 3.

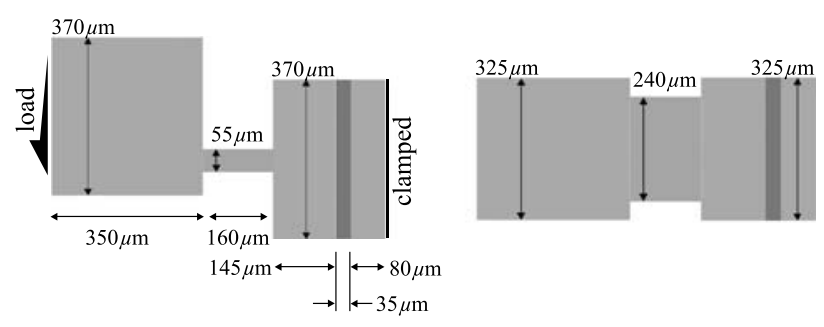

FIG. 7. Dimensions of pedicle-and-joint model shown in Figure 6. On the left is a side view; also indicated is the load applied to the left-hand face of the block corresponding to the long process, and the clamping of the right-hand face of the block corresponding to the head of the stapes. On the right is a top view. The thickness of the joint capsule (not shown) is $30 \mu \mathrm{m}$.

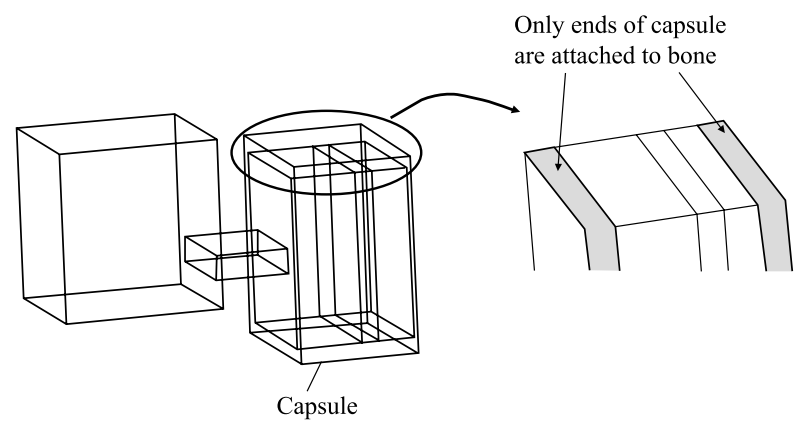

FIG. 8. Diagram showing how only the ends of the joint capsule are attached to the rest of the model. On the left is a wireframe version of the model with the joint capsule in place. On the right is a drawing of part of the model with the joint capsule removed; the grey bands indicate where the joint capsule is attached to the bone of the lenticular plate (left) and stapes head (right).

The finite-element mesh was generated via GiD (http:// gid.cimne.upc.es) using tetrahedral elements. (Hexahedral elements could easily have been used, in view of the simple geometry of the present model, but the use of tetrahedra provides experience that will be applicable when future models are developed with more irregular shapes.)

In order to determine a suitable mesh resolution, we performed convergence tests on most of the substructures in the model with both compressive and shearing loads. An optimal resolution was then decided upon for each substructure. To keep the number of nodes reasonably small, we allowed a discrepancy of up to $30 \%$ between simulation result and theoretical result for simple geometries, considering this to be acceptable in view of the uncertainties of the Young's moduli of each substructure. The final mesh is shown in Figure 9.

The finite-element program used was SAP IV (Bathe et al. 1974). This is the same software as that used in our previous modeling (e.g., Funnell et al. 1987), except that we are currently using it under

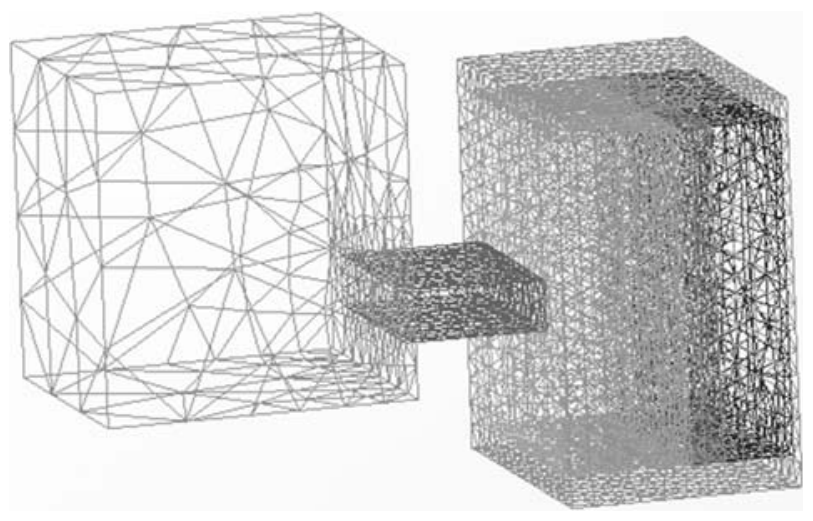

FIG. 9. Tetrahedral finite-element mesh of the model. 
Debian GNU/Linux, with either Intel $x 86$ or HP Alpha hardware. The mesh output by GiD was converted to the format required by SAP IV via a small locally developed program.

\section{Material properties}

The material properties of the model are assumed to be linear, uniform, and isotropic. The assumption of linearity is generally valid in the middle ear under normal hearing conditions; the assumptions of uniformity and isotropy are first-order approximations. For simplicity, the frequency is assumed here to be low enough that the effects of damping and inertia are negligible; this means that the material properties of a linear isotropic structure can be completely described by its Young's modulus and its Poisson's ratio. Poisson's ratio for all materials has been taken to be 0.3 . Explicit sensitivity tests suggest that varying the Poisson's ratio has little effect on the simulation results of the model, which is consistent with the findings of Funnell (1975).

In the following sections, the values of Young's moduli adopted for various parts of the model are explained.

Pedicle. Evidence from the serial histological sections clearly demonstrates that the pedicle is a continuation of bone from the long process of the incus to the lenticular plate. The Young's modulus for bone varies from 1 to $27 \mathrm{GPa}$, depending on the nature of the bone, the direction of measurement, and the part of the bone.

For estimating the stiffness of the pedicle, the work performed by Mente and Lewis (1994) is particularly useful because the measurements were conducted on small bone specimens obtained beneath joint cartilage. Because the pedicle is very close to the joint cartilage, it can be considered subchondral bone, and therefore a Young's modulus of $5 \mathrm{GPa}$ (Mente and Lewis 1994) is adopted for the pedicle.

The histological sections suggest, however, that the pedicle is comparable to a single hemi-osteon. Ascenzi and Bonucci (1967) calculated a Young's modulus of 10.7 GPa for a single osteon. Rho et al. (1998) calculated a modulus of $21.7 \mathrm{GPa}$ but the value might have been overestimated because the bone specimens used were dehydrated, which can lead to an increase in stiffness (Elices 2000). Therefore the simulations also include cases in which a Young's modulus of $12 \mathrm{GPa}$ is used for the pedicle.

Joint. The incudostapedial joint is a synovial joint, in which the load is transferred from a cartilage layer on one bone to a cartilage layer on the other bone, either through direct contact or through a thin film of synovial fluid between the cartilage layers, or by a mixture of both. Examination of the histological images shows that the space between the two articulating surfaces is so narrow that the cartilage on both sides is probably (at least partially) in direct contact during acoustic vibration. Therefore the articulation of the joint has been modeled as a single block of articular cartilage (ignoring the synovial fluid space). The cartilage is given a Young's modulus of $10 \mathrm{MPa}$, approximately the value measured in normal human articular cartilage (Elices 2000).

The synovial fluid functions as a lubricant, which allows the two articulating surfaces to glide easily. The modeling of the thin film of synovial fluid would be technically complicated and has not been implemented in this model. This implies that the two articulating surfaces in the model are firmly attached and the three components of the stress are transferred, with no loss, from one surface to another in the joint. In reality, the stress that is parallel to the articulating surface may be greatly attenuated because of the synovial fluid. As a consequence, the inplane displacements of the footplate may be smaller than those predicted by the simulation results.

Joint capsule. The incudostapedial joint-capsule ligament has been found to consist mainly of elastin (Davies 1948; Harty 1953), rather than collagen as found in most joint capsules. The Young's modulus of elastin fibers from bovine ligament has been reported as $1.1 \mathrm{MPa}$ (e.g., Gosline et al. 2002). A value of $1 \mathrm{MPa}$ has been adopted here for the joint capsule. The Young's modulus might be higher if there is a significant amount of collagen among the elastin.

Long process of incus. The long process of the incus is given a Young's modulus of $12 \mathrm{GPa}$, corresponding to stiff compact bone. Because the long process of the incus is so wide that it will bend little, the exact value of its Young's modulus is unimportant.

Lenticular plate and stapedial head. The lenticular plate and the head of the stapes consist predominantly of a combination of calcified cartilage and subchondral bone, the Young's moduli of which are 0.3 and $5 \mathrm{GPa}$, respectively (Mente and Lewis 1994). Hence an intermediate value of $1 \mathrm{GPa}$ is used here as the Young's modulus for the two structures.

\section{Applied load and boundary conditions}

For the isolated pedicle-and-joint model described above, the applied load was taken to be a downward shearing force uniformly applied to the cut surface of the long process of the incus (as shown in Fig. 7). The surface of the head of the stapes opposite the joint (where the crura would be) was clamped. This particular load was chosen specifically to induce bending in the pedicle. 


\section{Overall middle-ear model}

The simple applied load and boundary conditions described above clearly may not be representative of what really happens in the middle ear as the 3-D vibrations of the eardrum, malleus, and incus drive the pedicle, and as the stapes footplate acts in the oval window. To obtain more realistic conditions, for some simulations we embedded the pedicle-and-joint model in a complete middle-ear model.

The geometry of the middle-ear model was based in part on a 3-D reconstruction created by us from magnetic resonance microscopy (MRM) data (Henson and Henson, dataset 516871), and in part on a previous finite-element model (Funnell 1996). Figure 10 shows the MRM-based model. The geometry of the previous finite-element model, which had been based on histological serial sections, was modified to match the MRM-based model. The finite-element model includes shell representations of the eardrum and ossicles, and springs representing the middle-ear ligaments and cochlear load. The model of the eardrum is essentially the same as in previous models (Funnell et al. 1987, 1992), the pars tensa is assigned a Young's modulus of $20 \mathrm{MPa}$, a Poisson's ratio of 0.3 , and an overall thickness of 40 $\mu \mathrm{m}$. The footplate annular ligament and the cochlear load together are represented by 40 out-of-plane springs, each having a stiffness of $11 \mathrm{~N} / \mathrm{m}$, and $40 \mathrm{in}-$ plane springs, each having a stiffness of $9.9 \mathrm{~N} / \mathrm{m}$ (Ladak 1993, Ladak and Funnell 1994, 1996). The connections of the malleus and incus to the middleear cavity walls are represented by springs (Funnell 1996); the lateral bundle of the posterior incudal ligament is represented by two springs with a total

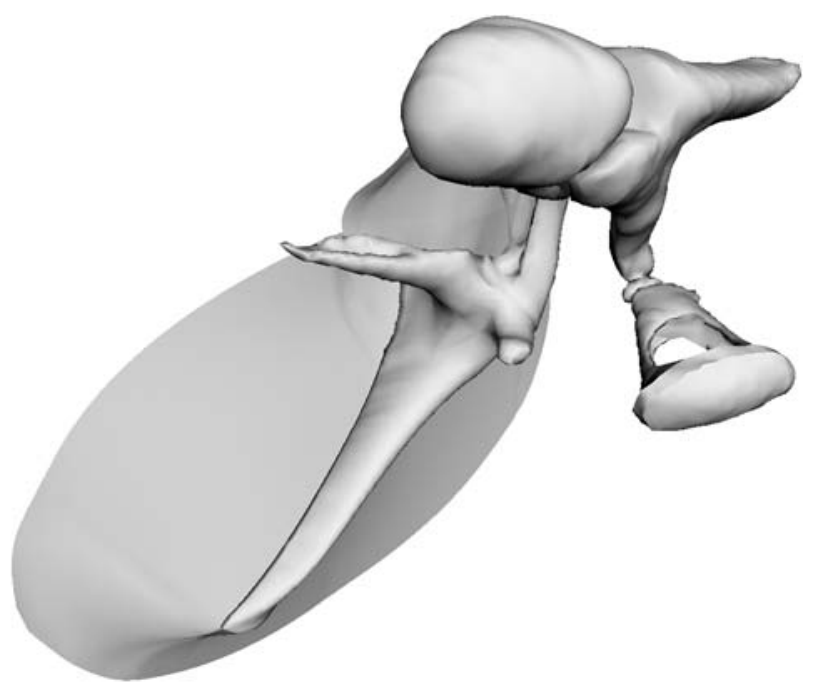

FIG. 10. Model of cat ossicles and tympanic membrane, based on magnetic resonance microscopy data.

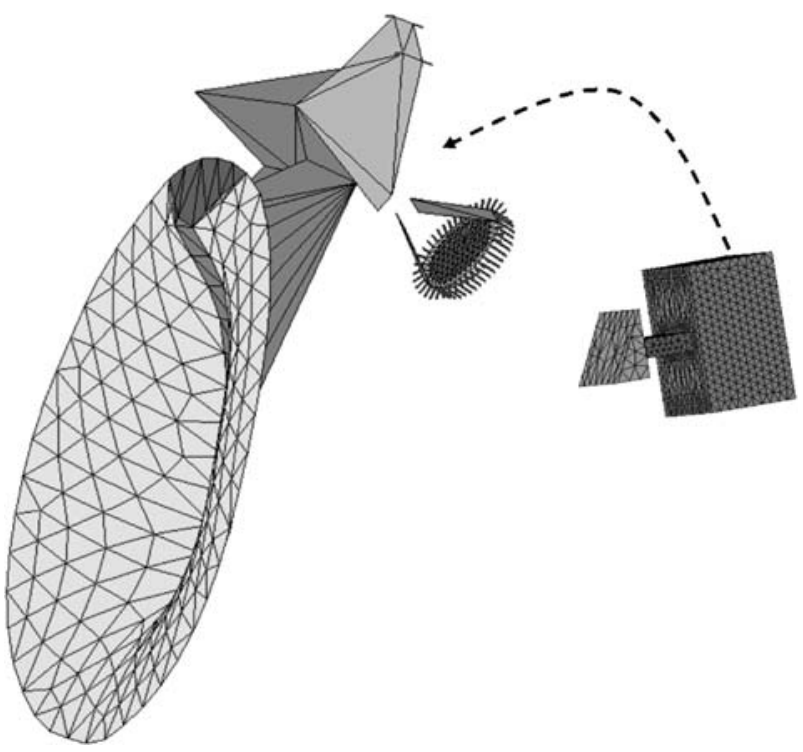

FIG. 11. Embedding of pedicle-and-joint model in overall middleear model.

stiffness of $10 \mathrm{kN} / \mathrm{m}$, the medial bundle by two springs with a total stiffness of $30 \mathrm{kN} / \mathrm{m}$, and the attachment of the malleus by three widely spaced springs with a total stiffness of $4 \mathrm{kN} / \mathrm{m}$. The simple incudostapedial joint model of Funnell (1996), which was based on that of Ghosh and Funnell (1995), is removed and replaced by the new pedicleand-joint model. Figure 11 shows the middle-ear model and how the pedicle-and-joint model is inserted. The block of the pedicle-and-incus model corresponding to the long process of the incus is reshaped to facilitate its attachment to the middleear model; because the block is very thick and stiff, it is practically rigid and its reshaping has essentially no effect on the behavior of the rest of the model.

The stimulus applied to this model is a uniform low-frequency acoustical pressure across the eardrum.

\section{RESULTS}

\section{Isolated model}

Figure 12 shows the displacements of the isolated pedicle-and-joint model with the base model parameters as established above. (Model displacements in the linear range are extremely small. In Figures 12 and 13, the displacements have been scaled up to make them visible.) It can be seen that the bending of the bony pedicle is quite considerable. In order to quantify the relative amounts of bending due to the joint and to the pedicle, the rotational displacements $\alpha$ (joint) and $\beta$ (pedicle) were computed, as 


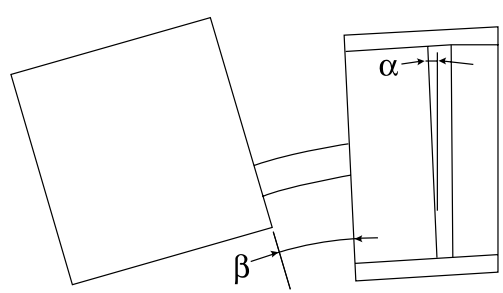

FIG. 12. Simulation results for isolated pedicle-and-joint model for base case: Young's moduli for pedicle, articulation and joint capsule are $5 \mathrm{GPa}, 10 \mathrm{MPa}$ and $50 \mathrm{MPa}$, respectively. $\alpha$ and $\beta$ are the rotational displacements presented in Tables 1 and 2.

indicated in the figure. For this base set of parameter values, $\alpha=4.4 \mu \mathrm{rad}$ and $\beta=11.6 \mu \mathrm{rad}$; that is, the pedicle contributes more than twice as much flexibility as does the incudostapedial joint itself.

In view of the uncertainties in the values of the various Young's moduli, simulations were performed for various values of the Young's moduli corresponding to the pedicle, articulation, and joint capsule, as summarized in Table 1. For each set of parameter values, the resulting values of $\alpha$ (joint bending), $\beta$ (pedicle bending), $\alpha+\beta$ (total bending), and $\beta / \alpha$ are given. Row $a$ corresponds to the base set of values.

In rows $b$ to $e$ the Young's modulus of the pedicle is left at its base value and the Young's moduli of the articulation and joint capsule are varied. For these cases, the pedicle bending $(\beta)$ is almost constant at 11.6-11.7. In row $b$, the flexibility of the joint increases because the Young's modulus of the articulation is reduced from 10 to $5 \mathrm{MPa}$; the value of $\alpha$ increases by a factor of 2 as the stiffness of the articulation is decreased by a factor of 2 . In rows $c$ and $d$, the Young's modulus of the joint capsule was reduced from 1 to $0.5 \mathrm{MPa}$, and increased to $2 \mathrm{MPa}$, respectively. For row $c$ the halving of the capsule stiffness leads to a $2 \%$ increase of $\alpha$, while for row $d$ the doubling of the capsule stiffness results in a $3 \%$ decrease of $\alpha$; evidently, the capsule is so flexible that it has little effect. In row $e$, the Young's moduli of the articulation and the capsule were both reduced, to values of 5 and $0.5 \mathrm{MPa}$, respectively; the value of $\alpha$ more than doubles. The flexibility of the joint is greatest in this case but $\beta$ is still greater than $\alpha$.

In rows $f$ and $g$, the Young's moduli for the articulation and joint capsule are left at their base values and the Young's modulus of the pedicle is varied. In row $f$, the pedicle stiffness was increased from 5 to $12 \mathrm{GPa}$, to equal that of the long process of the incus; as the pedicle becomes stiffer, the pedicle bending decreases by $36 \%$ but it is still about 1.7 times as large as that of the joint. In row $g$, the Young's modulus of the pedicle was reduced from 5 to $3 \mathrm{GPa}$; the pedicle bending increases by $40 \%$ and this case results in a significant increase of the ratio $\beta / \alpha$ and in the highest value of $\alpha+\beta$, the overall bending of the pedicle-and-joint model.

In summary, even if the pedicle is made much stiffer (row $f$ ) or the joint is made much less stiff (row $e$ ), the pedicle flexes more than the joint does.

\section{Combined model}

The overall middle-ear model presented here results in displacements of 308,135 , and $11.3 \mathrm{~nm} / \mathrm{Pa}$ for the eardrum (maximal displacement), umbo, and stapes footplate, respectively. These values can be compared with experimentally determined values of about $100 \mathrm{~nm} / \mathrm{Pa}$ for the eardrum, $40-50 \mathrm{~nm} / \mathrm{Pa}$ for the umbo (e.g., Decraemer et al. 1989), and about $30 \mathrm{~nm} / \mathrm{Pa}$ for the stapes footplate (Decraemer et al. 2000). The simulated displacements are each within about a factor of 3 of the experimental measurements. This is probably close enough for the present purpose, which is simply to evaluate the relative stiffnesses of the pedicle and joint with applied forces and loads which are somewhat more realistic than those in the isolated model.

\section{TABLE 1}

Simulation results for isolated pedicle-and-joint model, for various values of the Young's moduli of the pedicle, articulation and joint capsule

\begin{tabular}{|c|c|c|c|c|c|c|c|}
\hline & Pedicle (Pa) & Articulation (Pa) & Capsule (Pa) & $\alpha(\mu \mathrm{rad})$ & $\beta(\mu r a d)$ & $\alpha+\beta(\mu \mathrm{rad})$ & $\beta / \alpha$ \\
\hline $\mathrm{a}$ & $5 \mathrm{G}$ & $10 \mathrm{M}$ & $1 \mathrm{M}$ & 4.45 & 11.56 & 16.01 & 2.60 \\
\hline b & $5 \mathrm{G}$ & $5 \mathrm{M}$ & $1 \mathrm{M}$ & 8.90 & 11.72 & 20.62 & 1.32 \\
\hline c & $5 \mathrm{G}$ & $10 \mathrm{M}$ & $0.5 \mathrm{M}$ & 4.53 & 11.60 & 16.13 & 2.56 \\
\hline$d$ & $5 \mathrm{G}$ & $10 \mathrm{M}$ & $2 M$ & 4.32 & 11.64 & 15.96 & 2.69 \\
\hline e & $5 \mathrm{G}$ & $5 \mathrm{M}$ & $0.5 \mathrm{M}$ & 9.13 & 11.64 & 20.77 & 1.28 \\
\hline f & $12 G$ & $10 \mathrm{M}$ & $1 \mathrm{M}$ & 4.45 & 7.39 & 11.84 & 1.66 \\
\hline$g$ & $3 G$ & $10 \mathrm{M}$ & $1 \mathrm{M}$ & 4.45 & 16.20 & 20.66 & 3.64 \\
\hline
\end{tabular}

$\alpha$ and $\beta$ are the rotational displacements of the joint and pedicle, respectively; $\alpha+\beta$ gives the total bending and $\beta / \alpha$ gives the ratio of the two components of bending. Row a represents the base case. Rows $b$ to e correspond to variations of the joint stiffnesses, and rows $f$ and $g$ correspond to variations of the pedicle stiffness. The Young's moduli shown in bold are those which are different from the base case. 
TABLE 2

Simulation results for pedicle-and-joint model embedded within overall middle-ear model, for various values of the Young's moduli of the pedicle, articulation and joint capsule, as in Table 1

\begin{tabular}{|c|c|c|c|c|c|c|c|}
\hline & Pedicle (Pa) & Articulation (Pa) & Capsule (Pa) & $\alpha$ ( $\mu r a d)$ & $\beta$ ( $\mu$ rad) & $\alpha+\beta(\mu \mathrm{rad})$ & $\beta / \alpha$ \\
\hline a & $5 \mathrm{G}$ & $10 \mathrm{M}$ & $1 \mathrm{M}$ & 2.41 & 8.74 & 11.15 & 3.64 \\
\hline b & $5 \mathrm{G}$ & $5 \mathrm{M}$ & $1 \mathrm{M}$ & 4.43 & 7.94 & 12.37 & 1.79 \\
\hline C & $5 \mathrm{G}$ & $10 M$ & $0.5 \mathrm{M}$ & 2.43 & 8.81 & 11.24 & 3.62 \\
\hline d & $5 \mathrm{G}$ & $10 \mathrm{M}$ & $2 M$ & 2.35 & 8.80 & 11.15 & 3.74 \\
\hline $\mathrm{e}$ & $5 \mathrm{G}$ & $5 \mathrm{M}$ & $0.5 \mathrm{M}$ & 4.51 & 7.83 & 12.34 & 1.73 \\
\hline$f$ & $12 \mathrm{G}$ & $10 \mathrm{M}$ & $1 \mathrm{M}$ & 2.54 & 7.23 & 9.77 & 2.85 \\
\hline g & $3 \mathrm{G}$ & $10 \mathrm{M}$ & $1 \mathrm{M}$ & 2.24 & 10.07 & 12.31 & 4.49 \\
\hline
\end{tabular}

Table 2 shows the bending of the pedicle and joint for various values of the Young's moduli, when the pedicle-and-joint model is embedded in the overall middle-ear model. The columns and rows of the table are arranged the same as in Table 1. It can be seen that the pedicle bends more than three times as much as the joint itself for the base set of parameters (row $a$ ). In the extreme case (row $g$ ), the pedicle bends more than four times as much as the joint. Even when the joint is at its most flexible (row $e$ ), the pedicle bends almost twice as much as the joint.

Figure 13 shows the displacements of the pedicle and joint when embedded in the overall middle-ear model, for the base set of parameters. It can be seen that the more realistic load conditions result in a significant twisting of both the pedicle and the joint, in addition to the bending seen in the simple isolated model.

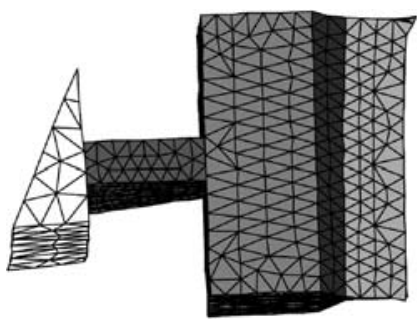

a

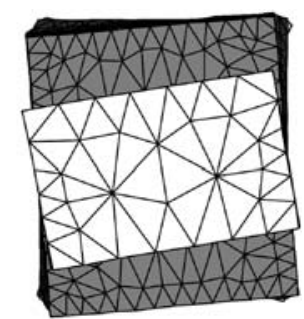

b
FIG. 13. Simulation results for pedicle-and-joint model when combined with overall middle-ear model, for base set of parameters. (a) The block corresponding to the displaced stapes head (far right) has been oriented in approximately the same way as in Figure 12. (b) The model in (a) has been rotated by $90^{\circ}$ so that the incus block is in front. In addition to the type of bending seen in Figure 12, there is also a twisting of the pedicle and joint about an axis running from the long process of the incus to the head of the stapes. (The 'ears' projecting from the four corners of the rightmost surface are small artefacts due to the way the stapes-head part of the pedicle-and-joint model was connected to the crura of the overall middle-ear model.)

\section{DISCUSSION}

On the one hand, the pedicle is extremely fine, and therefore easy to miss in histological sections unless every section is kept and examined. As a result, in spite of clear demonstrations by Shrapnell (1832) and Eysell (1870) of a continuous bony pedicle joining the lenticular plate to the long process of the incus in humans, there have been continuing occasional statements that the lenticular plate is a separate bone. On the other hand, the structures are so small, and are surrounded by so much soft tissue, that the delicacy of the pedicle is very easy to miss clinically and during dissection. As a result, most drawings of the ossicles include little detail about the attachment of the lenticular plate to the incus and imply that the pedicle is more robust than it really is.

A more detailed appreciation of this region has direct clinical relevance. For example, the mechanical fragility of the pedicle may explain why this is the most commonly affected area during traumatic ossicular dislocation. Furthermore, this fragility needs to be kept in mind during otosclerosis surgery. With a Lippy or a Robinson prosthesis that attaches to the lenticular process, for example, a large proportion of stapedectomy revision cases for postoperative conductive hearing loss is caused by erosion of the incudal long process. The anatomy of the region should also be kept in mind when utilizing wire prostheses that are crimped onto the long process of the incus - such crimping may reduce the blood supply coming from the body of the incus, increasing the risk of incus necrosis and leading to prosthesis displacement.

The nature of the bony pedicle may also be important in modeling the mechanics of the ossicular chain. The model results presented here suggest that the pedicle may bend significantly, and may even provide more flexibility than the actual incudostapedial joint does to the coupling between the incus and the stapes. This model-based prediction of significant bending of a bony part of the middle ear is reminis- 
cent of the prediction of manubrial bending (Funnell et al. 1992).

The nature of the flexibility of the pedicle is probably significantly different from that of the joint. Viewed along a line perpendicular to its bearing surface, the incudostapedial joint appears to be more or less circularly symmetric; in response to forces perpendicular to that line of view, it is presumably equally flexible in all directions. The bony pedicle, however, is very much thinner in one direction than in the other. Because of this, it may provide flexibility primarily in a single direction like a hinge, albeit with some additional twisting. It seems, in fact, to be well positioned to convert rotational motion of the incus into translational motion of the stapes, thus providing needed flexibility while retaining some control over the mode of vibration of the stapes. It may turn out to be worthwhile to attempt to replicate this behavior in middle-ear prostheses.

Further work is required to refine the shape of the model; a 3-D reconstruction is being developed based on our $1-\mu \mathrm{m}$ histological sections. In addition, the simulations need to be extended to higher frequencies. Such a model could be compared with experimental vibration measurements in cat, made on the long process of the incus, over the pedicle and lenticular plate, and on the head of the stapes. It will also be important to explore the extent and effects of anatomical variability of the middle-ear ossicles, and to study different species, including human.

\section{ACKNOWLEDGMENTS}

This work was supported by the Canadian Institutes of Health Research and the Natural Sciences and Engineering Research Council (Canada). Two of the authors (W.R.J.F. and M.D.M.) are members of the McGill Centre for Bone and Periodontal Research. We thank S.M. Khanna (Columbia University) for providing the paraffin histological sections, and I. Turgeon (McGill) for performing the plastic sectioning. We also thank M.M. Henson and O.W. Henson Jr. (UNC-Chapel Hill), and the Center for In Vivo Microscopy (Duke University) for the data used for the MRM-based model, and for valuable discussions of the anatomy of the lenticular process. Finally, we thank J. Lauzière for editing the manuscript.

\section{REFERENCES}

Ascenzi A, Bonucci E. The tensile properties of single osteons. Anat. Rec. 158(4):375-386, 1967.

Asherson N. The fourth auditory ossicle: fact or fantasy? J. Laryngol. Otol. 92(6):453-465, 1978.

Bathe K-J, Wilson EL, Peterson FE. SAP IV. A structural analysis program for static and dynamic response of linear systems.
Report No. EERC 73-11. University of California, Berkeley, vii +59 pp + appendices, 1974.

Browne T (ca. 1650). In: Keynes G (ed) The Works of Sir Thomas Browne, vol. III. University Press, Oxford, p 335, 1964.

DAVIES DV. A note on the articulations of the auditory ossicles. J. Laryngol. Otol. 62:533-538, 1948.

Decraemer WF, Khanna SM, Funnell WRJ. Interferometric measurement of the amplitude and phase of tympanic membrane vibrations in cat. Hear. Res. 38(1/2):1-17, 1989.

Decraemer WF, Khanna SM, Funnell WRJ. Measurement and modelling of the three-dimensional vibration of the stapes in cat. Proc. Internat. Symp. on Recent Developments in Auditory Mechanics, Sendai, Japan, 1999 July. World Scientific Pub., US, pp 36-43, 2000.

Doran AHG. Morphology of the mammalian ossicula auditûs. Trans. Linnean Soc. London 2nd series vol. I, 1879:371-497 + plates 58-64, 1878.

ELICES M. Structural Biological Materials. Pergamon, Amsterdam, $\mathrm{xv}+361 \mathrm{pp}, 2000$.

Eysell A. Beiträge zur Anatomie des Steigbügels und Seiner Verbindungen. Arch. Ohrenheilkund. 5:237-249+2 plates, 1870.

FunNell WRJ. A theoretical study of eardrum vibrations using the finite-element method. Ph.D. thesis, McGill University, Montréal, 1975.

FunNELL WRJ. Finite-element modelling of the cat middle ear with elastically suspended malleus and incus. 19th Midwinter Res. Mtg., Assoc. Res. Otolaryngol. 1996.

Funnell WRJ, Decraemer WF, Khanna SM. On the damped frequency response of a finite-element model of the cat eardrum. J. Acoust. Soc. Am. 81(6):1851-1859, 1987.

FunNell WRJ, Khanna SM, Decraemer WF. On the degree of rigidity of the manubrium in a finite-element model of the cat eardrum. J. Acoust. Soc. Am. 91(4):2082-2090, 1992.

GHosh SS, FunNell WRJ. On the effects of incudostapedial joint flexibility in a finite-element model of the cat middle ear. IEEE Eng. Med. Biol. Soc. 17th Ann. Conf., pp 1437-1438, 1995.

Gosline J, Lillie M, Carrington E, Guerette P, Ortlepp C, Savage K. Elastic proteins: biological roles and mechanical properties Philos. Trans. R. Soc. Lond. B 357(1418):121-132, 2002.

Harty M. Elastic tissue in the middle-ear cavity. J. Laryngol. Otol. 67:723-729, 1953.

Henson, JR OW. Some morphological and functional aspects of certain structures of the middle ear in bats and insectivores Univ. Kans. Sci. Bull. 42(3):151-255, 1961.

HÜtTEnBRink K-B. The middle ear as a pressure receptor. Middle ear mechanics in research and otosurgery. In: Hüttenbrink, K-B (ed) Proc. Internat. Workshop, Dresden, 1996 Sep 19-22. Dept. Oto-rhinolaryngol., Univ. Hosp. Carl Gustav Carus, Dresden Univ. Technology, pp 15-20, p 259, 1997.

HYRTL J. Vergleichend-anatomische Untersuchungen über das innere Gehörorgan des Menschen und der Säugethiere. Verlag von Friedrich Ehrlich, Prague, viii +139 pp + 9 plates, 1845 .

LADAK HM. Finite-element modelling of middle-ear prosthesis in cat. M. Eng. thesis, McGill University, Montréal, 1993.

LADAK HM, FunNell WRJ. Finite-element modelling of malleusstapes and malleus-footplate prostheses in cat. 17th Midwinter Res. Mtg., Assoc. Res. Otolaryngol., St. Petersburg Beach, 1994.

LADAK HM, FunNeLL WRJ. Finite-element modeling of the normal and surgically repaired cat middle ear. J. Acoust. Soc. Am. 100(2):933-944, 1996.

Mente PL, Lewis JL. Elastic modulus of calcified cartilage is an order of magnitude less than that of subchondral bone. J. Orthop. Res. 12:637-647, 1994.

Palchun VT, Magomedov MM. Some anatomical features of the long process of the incus. Vestn. Otorinolaringol. 2:19-20, 1997. 
Rho JY, Tsui TY, Pharr GM. Elastic properties of osteon and trabecular bone measured by nanoindentation. J. Biomed. Mater. Res. 45:48-54, 1998.

Shrapnell HJ. On the structure of the os incus. Lond. Med. Gaz. 171-173, 1832
Wolff D, Bellucci RJ, Eggston AA. Microscopic Anatomy of the Temporal Bone. Williams \& Wilkins, Baltimore, ix + 414 pp, 1957.

Wolff D, Bellucci RJ, Eggston AA. Surgical and Microscopic Anatomy of the Temporal Bone. Hafner, New York, 579 pp, 1971. 